\title{
Quercetin-gavaging quashes anaphylaxis sequela in the peanut sensitised wistars
}

\author{
Lotfollah Behroo*, Farideh Shishehbor, Mehri Ghafouriyan Broujerdnia, Forough Namjouyan, \\ Seiyed-Mahmoud Latifi
}

From Food Allergy and Anaphylaxis Meeting 2014

Dublin, Ireland. 9-11 October 2014

\section{Background}

Regarding the large figures of patients affected with potentially life-threatening peanut allergy, the possibility of inevitable occurrence of unexpected exposures and, the paucity of promising acceptable immunotherapy approaches, more applicable and at the same time, safe remedial strategies are necessitated.

Quercetin, a nutritional panacea belonging to "Flavonol" sub-group of the flavonoid family, thanks to its antioxidant/free radical-scavenging, anti-inflammatory and other protective properties has been suggested for therapy of humankind allergic diseases and might be helpful for the treatment of food allergies.

\section{Objective}

The main aim of our study was to investigate the antiaanaphylactic characteristics of quercetin in a wistar-rat model of peanut allergy.

\section{Material and methods}

40 male wistar rats, 4-6 weeks old at study start, were sensitized with crude peanut herape in the presence of cholera toxin adjuvant. The sensitized rats in the treatment group $(\mathrm{n}=10)$, were treated intragastrically with quercetin, at a dose of $50 \mathrm{mg} / \mathrm{Kg}$.BW over a period of four weeks. Subsequently, some well-known anaphylaxis-determinant variables were measured.

\section{Results}

Following calculations, it was revealed that quercetin has sufficiently abrogated peanut-induced anaphylactic reactions. Accordingly, plasma histamine and total serum IgE levels had been lowered significantly, in treatment group ( $\mathrm{p}<0.000$ and $\mathrm{p}<0.000$, respectively). Additionally,

AJUMS, Ahvaz, Iran 